\title{
Three-Dimensional Architecture of Latent and Active Meprin B
}

\author{
M. T. Norcum ${ }^{*}$, K. B. Labat ${ }^{*}$, G. P. Bertenshaw ${ }^{* *}$, and J. S. Bond ${ }^{* *}$ \\ *Biochemistry Department, University of Mississippi Medical Center, Jackson, MS 39216 \\ ** Department of Biochemistry and Molecular Biology, The Pennsylvania State University College of \\ Medicine, Hershey, PA 17033
}

Meprins [1] are zinc metalloproteinases of the astacin family and metzincin superfamily. Meprin proteins are abundantly expressed in the kidney and intestine and have been biochemically characterized in human, rat and mouse tissues. There are two subunit types ( $\alpha$ and $\beta)$ that are evolutionarily-related, multidomain glycosylated polypeptides. The proteins are synthesized as latent disulfide-linked membrane-spanning oligomers, but can also be found in secreted forms. Meprin A is either a heterooligomer of $\alpha$ and $\beta$ subunits or an $\alpha$ homooligomer, while meprin $B$ is a homooligomer of $\beta$ subunits (Figure 1). Meprins can cleave basement membrane proteins, growth factors, and a variety of other bioactive peptides [2].

In the first structural study of these enzymes, we showed that recombinant active murine homooligomeric meprin A forms rings and flexible chains that are at least octamers [3]. In contrast, homooligomeric recombinant rat meprin $\mathrm{B}$ consistently exists as a dimer of $\beta$ subunits. As determined by MALDI-TOF mass spectroscopy, the mass of latent meprin B is $166 \mathrm{kDa}$, while that of the active form is $154 \mathrm{kDa}$. The active form is generated in vitro by limited trypsin digestion [2].

In this study, both latent and active recombinant rat meprin B have been viewed in deep uranyl acetate negative stain. The random conical tilt reconstruction scheme using SPIDER software [4] was applied to image pairs taken at $55^{\circ}$ or $0^{\circ}$ tilt with minimum dose focusing on a LEO 912 energyfiltered microscope at absolute magnification of 63000. Negatives were digitized at a resolution of $3.22 \AA /$ pixel on the image scale. Resolution limits are from the $50 \%$ cutoff of Fourier shell correlation between volumes calculated from half data sets. The active structure was calculated de novo from merged volumes from image averages, while that for the latent form was calculated de novo (not shown) and by using the active form as a reference. Figure 2 shows that the angular distribution derived from projection mapping of images is essentially complete for both structures.

The architectures of both latent and active meprin B are very similar (Figure 3). Each measures $c a$. $10 \times 15 \times 20 \mathrm{~nm}$ and has a large central cavity. The volumes are presented at thresholds chosen to maximize structural detail and each has been adjusted in accordance with their relative molecular masses. The two-fold symmetry expected for dimeric structures is most easily seen in the $0^{\circ}$ and $270^{\circ}$ views. The overlay view in Figure 4 emphasizes the similarity of the structures. However, even at the current resolution limits of $c a .27 \AA$, subtle changes between the volumes are observable. These may correspond to structural rearrangements resulting from activation.

[1] J. S. Bond and R. J. Beynon, Prot. Sci., 4 (1995) 1247-1261.

[2] G. P. Bertenshaw et al., J. Biol. Chem., 276 (2001) 13248-13255.

[3] F. T. Ishmael et al., J. Biol. Chem., 276 (2001) 23207-23211.

[4] J. Frank et al., J. Struct. Biol., 116 (1996) 190-199.

[5] This work was supported in part by a fellowship from AHA (GPB) and grants from NIH (JSB). 


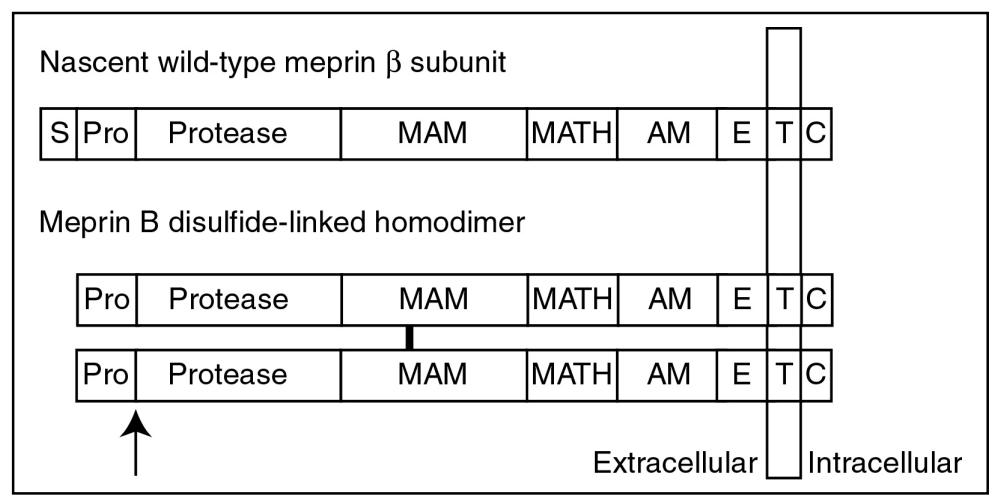

Fig 1. Domain and schematic dimeric structure of meprin B: signal peptide (S); propeptide (Pro); astacin-like catalytic domain (protease); meprin, A5 protein and protein tyrosine phosphatase $\mu$ (MAM); meprin and tumor necrosis factor receptor-associated factor homology domain (MATH); after MATH domain (AM); epidermal growth factor-like domain $(\mathrm{E})$; transmembrane spanning region $(\mathrm{T})$; and cytoplasmic tail (C). Arrow indicates activation cleavage site.

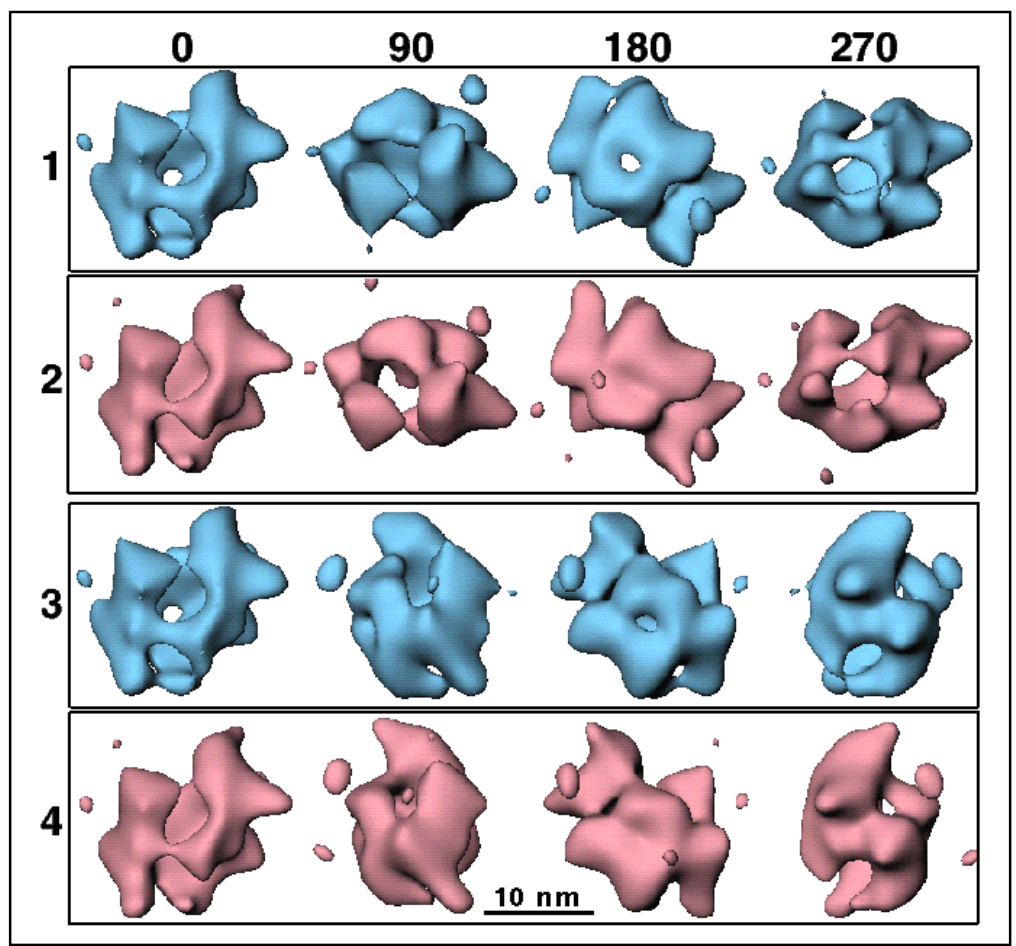

Fig 3. Three-dimensional volumes of latent (Rows 1 and 3, blue) and active meprin B (Rows 2 and 4, pink) showing successive $90^{\circ}$ rotations around their horizontal (Rows 1 and 2) and vertical axes (Rows 3 and 4).
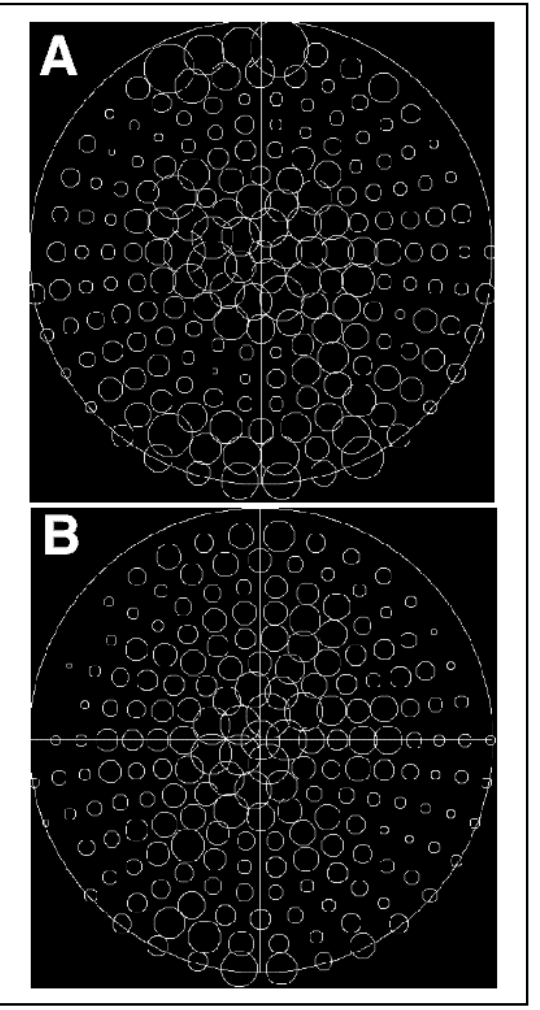

Fig 2. Angular distribution of images from which the volumes have been calculated for latent (Panel A) and active meprin B (Panel B).

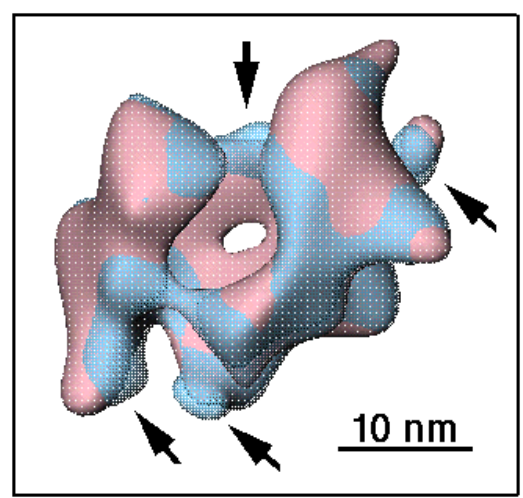

Fig. 4. Overlay of threedimensional volumes of latent (transparent blue) and active (pink) meprin B shows subtle structural changes (arrows) at ca. $27 \AA$ resolution. 